\title{
Rang de familles de courbes elliptiques
}

\author{
par

\section{Odile Lecacheux (Paris)}

1. Introduction. Soit $E$ une courbe elliptique sur $\mathbb{Q}$. Le théorème de Mordell-Weil donne la structure du groupe des points rationnels de $E$ :

$$
E(\mathbb{Q}) \simeq E(\mathbb{Q})_{\text {tors }} \times \mathbb{Z}^{r}
$$

où $r$ est appelé le rang sur $\mathbb{Q}$ de $E$. Un théorème de Mazur donne les seules structures de torsion possibles :

$$
E(\mathbb{Q})_{\text {tors }}= \begin{cases}\mathbb{Z} / m \mathbb{Z}, & m=1,2, \ldots, 10,12, \\ \mathbb{Z} / 2 \mathbb{Z} \times \mathbb{Z} / 2 m \mathbb{Z}, & m=1, \ldots, 4 .\end{cases}
$$

On notera $\mathcal{E}_{N}$ (resp. $\mathcal{E}_{n, m}$ ) l'ensemble des courbes elliptiques sur $\mathbb{Q}$ telles que $E(\mathbb{Q})_{\text {tors }} \simeq \mathbb{Z} / N \mathbb{Z}$ (resp. $E(\mathbb{Q})_{\text {tors }} \simeq \mathbb{Z} / n \mathbb{Z} \times \mathbb{Z} / m \mathbb{Z}$ ), et on définit

$$
\begin{aligned}
b(N) & =\sup \left\{r ; E \in \mathcal{E}_{N}\right\}, & B(N) & =\lim \sup \left\{r ; E \in \mathcal{E}_{N}\right\}, \\
b(n, m) & =\sup \left\{r ; E \in \mathcal{E}_{n, m}\right\}, & B(n, m) & =\lim \sup \left\{r ; E \in \mathcal{E}_{n, m}\right\} .
\end{aligned}
$$

On ignore si $b(n, m)$ et $b(N)$ sont finis. Sans entrer dans les détails il faut retenir les résultats suivants :

- $b(0) \geq 24$ et $B(0) \geq 14$,

- $B(N)$ et $B(n, m) \geq 1$ pour toutes les valeurs de $N, n$ et $m$, par exemple $B(2) \geq 8, B(5) \geq 3$.

Citons les travaux de divers auteurs qu'on pourrait presque faire remonter à Fermat : Kretschmer, Nagao, Fermigier, Mestre, Martin-McMillen, Kihara, Kulesz, Atkin-Morain, Dujella ... et d'autres (voir [3]).

Dans cet article nous nous intéresserons à $B(N)$ pour $N=7$. Pour cet entier des exemples ont été construits et donnent $B(7) \geq 1$ (Kulesz [4], Atkin-Morain [1]). Notons aussi que $b(7) \geq 5$ [3].

Nous obtenons les résultats suivants :

THÉORÈme 1. L'entier B(7) est supérieur ou égal à 2.

2000 Mathematics Subject Classification: Primary 11G05, 14H52; Secondary 14H10, 14J27, 14J28. 
Plus précisément, il existe des courbes elliptiques sur $\mathbb{Q}$, de rang au moins deux sur $\mathbb{Q}$, ayant un point d'ordre 7 rationnel, paramétrées par les points rationnels de plusieurs courbes elliptiques dont au moins deux ont un rang sur $\mathbb{Q}$ égal à 3 . Parmi ces familles de courbes ainsi construites, ayant un point de 7 -torsion $\mathbb{Q}$-rationnel, certaines ont un rang 3.

2. Notations et méthode. On désignera par $N$ un entier $\geq 5$; on note $Y_{1}(N)$ la courbe modulaire sur $\mathbb{Q}$ qui paramétrise les couples $\left(E, A_{N}\right)$ où $E$ est une courbe elliptique, ayant un point $A_{N}$ d'ordre exactement $N$. Soit $X_{1}(N)$ la compactification de $Y_{1}(N)$.

Soit $E_{N}$ la courbe elliptique universelle qui correspond à cette structure. Alors $E_{N}$ est une surface elliptique définie sur $\mathbb{Q}$ avec un morphisme de projection

$$
\pi: E_{N} \rightarrow Y_{1}(N)
$$

et une section $s: Y_{1}(N) \rightarrow E_{N}$, tous deux définis sur $\mathbb{Q}$.

La courbe $X_{1}(7)$ est de genre 0 et on notera $d$ un générateur de son corps de fonctions. Nous noterons $E_{d}$ la fibre générique. Enfin nous noterons $\mathbb{P}_{z}^{1}$ l'espace projectif avec point générique $z$.

Pour $N=7$, le modèle minimal non singulier de la surface $E_{7}$ est une surface $K 3$ ([7, pp. 276-277]). Nous montrons que la surface $E_{7}$ est birationnellement équivalente à la surface $S_{7}$ d'équation

$$
(u v-u-v)(d v-1)(d u-1)=u v(u-1)(v-1) d(d-1)
$$

et nous construisons une autre fibration elliptique de la surface $S_{7}$,

$$
\phi_{v}: S_{7} \rightarrow \mathbb{P}_{v}^{1},
$$

avec une section définie sur $\mathbb{Q}$. Nous noterons $H_{v}$ la fibre générique. Les sections de $E_{7}$ correspondant aux points d'ordre 7 de $E_{d}$ donnent sur $H_{v}$ un point d'ordre 4 ainsi qu'un point $P$ d'ordre infini sur $\mathbb{Q}(v)$. On construit ainsi en considérant les multiples de $P$ une infinité de revêtements de

$$
\mathbb{P}_{v_{n}}^{1} \rightarrow \mathbb{P}_{d}^{1}
$$

et les changements de base $E_{d} \times_{\mathbb{P}_{d}^{1}} \mathbb{P}_{v_{n}}^{1}$ correspondants, ce qui donne une courbe elliptique de rang au moins 1 sur $\mathbb{Q}\left(v_{n}\right)$ avec un point de 7-torsion.

Parmi ces revêtements nous avons étudié ceux de petit degré, en particulier de degré 2 , correspondant aux fractions rationnelles $d_{0}, d_{1}, d_{4}$ et $d_{5}$.

En prenant le produit fibré de deux tels changements de base on obtient une famille de courbes elliptiques de rang au moins deux au-dessus d'une base de genre 1. Cette dernière courbe, dans les cas étudiés, est une courbe elliptique de rang au moins 1 sur $\mathbb{Q}$. On obtient ainsi une infinité de courbes de rang au moins 2 sur $\mathbb{Q}$ munies d'un point $\mathbb{Q}$-rationnel d'ordre 7. Certaines courbes correspondant à trois points rationnels de $\mathbb{P}_{v_{n_{i}}}^{1}$ pour $i=1,2,3$ 
donnent des exemples de courbes de rang 3 munies d'un point $\mathbb{Q}$-rationnel d'ordre 7 .

2.1. La surface $E_{7}$ et ses automorphismes. Soit $E$ une courbe elliptique sur $\mathbb{Q}$ ayant un point rationnel de 7 -torsion. Il existe un rationnel $d$ tel que la courbe $E$ soit $\mathbb{Q}$-isomorphe à la courbe $E_{d}$ d'équation

$$
\left(E_{d}\right): \quad y^{2}+\left(1+d-d^{2}\right) x y+\left(d^{2}-d^{3}\right) y=x^{3}+\left(d^{2}-d^{3}\right) x^{2}
$$

de discriminant $d^{7}(d-1)^{7}\left(d^{3}-8 d^{2}+5 d+1\right)$ et où le point d'ordre 7 est le point $A=(0,0)$. Si on considère $d$ comme une indéterminée on notera $E_{7}$ la surface d'équation

$$
y^{2}+\left(1+d-d^{2}\right) x y+\left(d^{2}-d^{3}\right) y=x^{3}+\left(d^{2}-d^{3}\right) x^{2} .
$$

La surface elliptique $E_{7}$ admet l'automorphisme d'ordre 6 noté $\sigma$ défini par

$$
\begin{array}{lll}
x \mapsto \frac{x-d^{2}(d-1)}{d^{4}}, & x \mapsto \frac{x+d-d^{2}}{(d-1)^{4}}, \\
y \mapsto \frac{-y-d x+d^{3}(d-1)}{d^{6}}, & \text { d'inverse } & y \mapsto \frac{-(x+y)+x d}{(d-1)^{6}}, \\
d \mapsto \frac{d-1}{d}, & d \mapsto \frac{-1}{d-1},
\end{array}
$$

qui a l'interprétation modulaire suivante : au couple $(E, A)$ on associe le couple $(E, i A)$ avec $(i, 7)=1$.

Si $M$ est un point générique de la courbe $E_{d}$, l'automorphisme défini sur $E_{d}$ par $M \mapsto A+M$ définit un automorphisme noté $\sigma_{7}$, d'ordre 7 , de $E_{7}$.

2.2. La surface $S_{7}$. Faisons les changements de coordonnées

$$
x=\frac{d(d-1)}{u+v-u v}, \quad y=\frac{(d-1)^{2} d^{2} u}{(u+v-u v)^{2}}
$$

d'inverse

$$
u=\frac{y}{x^{2}}, \quad v=\frac{(x+d) d(d-1)-(x+y)}{x^{2}} .
$$

Les fonctions $u$ et $v$ sur $E_{d}$ ont comme diviseur

$$
\operatorname{div}(u)=-2(6 A)+5 A+\infty, \quad \operatorname{div}(v)=-2(A)+2 A+\infty .
$$

La surface $E_{7}$ est birationnellement équivalente à la surface

$$
S_{7}: \quad-d(d-1) u v+(u v-u-v)(1+d(u v-u-v))=0 .
$$

Nous utiliserons aussi les deux factorisations suivantes de l'équation de $S_{7}$ :

$$
\begin{aligned}
(d v-1)(u v-u-v)(d u-1) & =u v(u-1)(v-1) d(d-1), \\
u(u-1) d(v-1)^{2} & =(d v-1)(u d+v u-u-v),
\end{aligned}
$$

ainsi que celle obtenue en intervertissant $u$ et $v$. 
On remarque que l'involution qui échange $u$ en $v$ correspond à l'involution $P \mapsto-P$ sur la courbe elliptique $E_{d}$.

L'automorphisme $\sigma$ est défini sur 2.1 par

$$
(u, v, d) \mapsto\left(\frac{(d v-1)(u v-u-v)^{2}}{(d-1)^{2} u v^{2}}, \frac{(d u-1)(u v-u-v)^{2}}{(d-1)^{2} v u^{2}}, \frac{d-1}{d}\right) .
$$

L'automorphisme $\sigma_{7}$ est défini sur 2.1 par

$$
(u, v, d) \mapsto\left(\frac{(u-1)(u v-u-v)}{d u^{2}(d-1)},-\frac{1}{d(u v-u-v)}, d\right) .
$$

Proposition 2. La fibration

$$
\phi: S_{7} \rightarrow \mathbb{P}_{v}^{1}, \quad(u, v, d) \mapsto v,
$$

définit sur $S_{7}$ une structure de surface elliptique de fibre générique $H_{v}$. La torsion du groupe de Mordell-Weil $H_{v}(\mathbb{Q}(v))$ est cyclique d'ordre 4 et le rang de ce groupe est égal à 1 .

On pose

$$
u=\frac{d v-1+Y}{d(v-1)}, \quad \text { soit } \quad Y=1+d(u v-u-v) .
$$

On obtient alors une cubique en $(Y, d)$ dépendant de $v$.

Les transformations habituelles pour obtenir une forme de Weierstrass sont

$$
\begin{aligned}
U & =v(v-1)\left(Y+d v-\left(v^{2}-v+1\right)\right), \\
u & =\frac{\left(U+v^{2}(v-1)^{2}\right) U}{Z(v-1)^{2}} .
\end{aligned}
$$

Le changement de variable

$$
\begin{aligned}
& d=\frac{Z}{v U}, \\
& x=\frac{(v-1) Z^{2}(U v-Z)}{v^{2} U^{2}\left(U^{2}+v^{2}(v-1)^{2} U-v Z(v-1)\right)}, \\
& y=\frac{Z^{3}(U v-Z)^{2}\left(U+v^{2}(v-1)^{2}\right)}{v^{4} U^{3}\left(U^{2}+v^{2}(v-1)^{2} U-v Z(v-1)\right)^{2}}
\end{aligned}
$$

donne un modèle de Weierstrass de la fibre $H_{v}$, soit

$$
\begin{aligned}
Z^{2}+v(2 v-3) U Z+ & v^{2}(2 v-1)(v-1)^{3} Z \\
& =U\left(U+v(v-1)\left(v^{2}-v+1\right)\right)\left(U+v^{2}(v-1)^{2}\right) .
\end{aligned}
$$

Les points d'ordre 7 de $E_{d}$ correspondent aux sections $U=Z$ et $v U=Z$, ce qui donne les points de $H_{v}$

$$
\begin{aligned}
& A_{1}=\left(U=-v^{2}(v-1)^{2}, Z=-v^{2}(v-1)^{2}\right), \\
& A_{2}=\left(-(v+1)(v-1)^{3},-(v+1)(v-1)^{3}\right),
\end{aligned}
$$




$$
\begin{aligned}
& A_{3}=\left(-v(v-1)^{3},-v^{2}(v-1)^{3}\right), \\
& A_{4}=\left(-v^{2}(v-1)(v-2),-v^{3}(v-1)(v-2)\right), \\
& A_{5}=(0,0) .
\end{aligned}
$$

Le point $A_{3}$ est d'ordre 2 et $2 A_{1}=A_{3}$, donc $A_{1}$ est d'ordre 4 . D'autre part on a les relations suivantes :

$$
\begin{aligned}
A_{1}+A_{5} & =A_{2}, \\
2 A_{1}+A_{5} & =A_{4}, \\
3 A_{1}+A_{5} & =\left(-v(v-1)\left(v^{2}-v+1\right), v^{2}(v-1)(v-2)\right) .
\end{aligned}
$$

En utilisant un résultat de Shioda [5], on peut calculer le rang sur $\mathbb{C}(v)$ de $H_{v}$. Par calcul on déduit le type des fibres dégénérées : en $v=0$ et $v=\infty$ les fibres sont de type $I_{1}^{*}$, en $v=1$ de type $I_{8}$ et en $v=(31 \pm 3 i \sqrt{7}) / 32$ de type $I_{1}$. Le nombre de composantes est respectivement $m_{s}=6,8$, et 1 . De la relation fondamentale

$$
\operatorname{Rang}\left(H_{v}(\mathbb{C}(v))\right)+2+\sum_{s}\left(m_{s}-1\right)=\operatorname{Rang} \operatorname{NS}\left(E_{7}\right)
$$

il résulte que le rang du groupe de Mordell-Weil de $H_{v}(\mathbb{C}(v))$ est inférieur ou égal à un, compte tenu de l'inégalité Rang $\mathrm{NS}\left(E_{7}\right) \leq 20$. Par spécialisation, par exemple pour $v=5$ on montre que le point $A_{5}$ spécialisé n'est pas d'ordre 2,8 ou 4, il est donc d'ordre infini compte tenu du théorème de Mazur.

On construit alors une infinité de familles à un paramètre de courbes elliptiques ayant de la 7 -torsion et un rang $\geq 1$. Pour cela on considère les points $r A_{1}+s A_{5}(s \in \mathbb{Z}, 0 \leq r \leq 3)$ de coordonnées $\left(u_{r, s}(v), v, d_{r, s}(v)\right)$ sur $S_{7}$. En utilisant le changement de coordonnées $(u, v) \mapsto(x, y)$ précédent on obtient la famille $\left(E_{d_{r, s}(v)}, P_{r, s}(v)\right)$. Montrons que $P_{r, s}(v)$ est d'ordre infini si $s \neq 0$. Si $r A_{1}+s A_{5} \neq A_{i}, 1 \leq i \leq 5$, le point $P_{r, s}(v)$ n'est pas un multiple de $A$. Il existe donc au moins une valeur de $v_{0} \in \mathbb{Q}$ pour laquelle le point $P_{r, s}\left(v_{0}\right)$ n'est pas multiple de $A$. Utilisant le théorème de Mazur, le point $P_{r, s}\left(v_{0}\right)$ ne peut être de torsion car la courbe $E_{d_{r, s}\left(v_{0}\right)}$ aurait un point rationnel d'ordre $7 m, m \neq 1$.

Si $d=P / Q$ où $P$ et $Q$ sont deux polynômes premiers entre eux, on pose $\mathrm{ht}(d)=\max (\operatorname{deg}(P), \operatorname{deg}(Q))$. Les familles correspondant aux points

$$
-2 A_{5}+h A_{1} \quad \text { avec } 0 \leq h \leq 4,
$$

vérifient $\operatorname{ht}(d)=2$.

2.3. Involutions. L'équation définissant $S_{7}$ est quadratique par rapport à chaque variable; la surface $S_{7}$ peut être considérée comme un revêtement double de $\mathbb{P}^{1} \times \mathbb{P}^{1}$ de 3 façons différentes, ce qui définit trois involutions 


$$
\begin{aligned}
e_{u}: \quad(u, v, d) & \mapsto\left(\frac{v(d v-1)}{d(v-1)^{2} u}, v, d\right), \\
e_{v}: \quad(u, v, d) & \mapsto\left(u, \frac{u(d u-1)}{d(u-1)^{2} v}, d\right), \\
e_{d}: \quad(u, v, d) & \mapsto\left(u, v,-\frac{(u-1)(v-1)(d-1)}{(d v-1)(d u-1)}\right) .
\end{aligned}
$$

On remarque que $\left(e_{u} \circ e_{v}\right)^{2}=\sigma_{7}$. Si $f=e_{u} \circ e_{v}, g=e_{d} \circ e_{v}$ et $f_{1}=g \circ f \circ g^{-1}$ alors $\sigma^{3}=f \circ f_{1} \circ f \circ e_{d}$ et $\sigma^{4}=\left(g^{2} \circ f \circ f_{1} \circ g^{2}\right)^{2}$. Un calcul montre que $e_{d} \circ e_{u}$ est d'ordre 4 , ainsi que $e_{d} \circ e_{v}$.

La surface $S_{7}$ contient les droites suivantes :

$$
\begin{array}{ll}
D_{0}: & u=0, v=0, \\
D_{1}: & d=1, u=1, \\
D_{2}: & d=1, v=1 .
\end{array}
$$

Le plan tangent à $S_{7}$ passant par $D_{1}$ a pour équation

$$
u+d=2 \text {. }
$$

La surface $S_{7}$ contient les courbes de genre 0 :

$$
\begin{array}{ll}
C_{1}: & d=0, u v-u-v=0 \\
C_{2}: & d=1, u v-u-v=0, \\
C_{3}: & u=0, d v=1 ; \quad \widetilde{C}_{3}: \quad u=1, d v=1 \\
C_{5}: & v=0, d u=1 ; \quad \widetilde{C}_{5}: \quad v=1, d u=1 \\
C_{6}: & u+d=2, d=-\frac{v-2}{v^{2}-v+1}, \\
C_{7}: & v+d=2, d=-\frac{u-2}{u^{2}-u+1} .
\end{array}
$$

La conique $C_{7}$ (resp. $\left.C_{6}\right)$ est stable par $e_{u}$ (resp. $\left.e_{v}\right)$.

Les points $-2 A_{5}+h A_{1}$ correspondent à $e_{u} \circ \sigma_{7}^{2} \circ\left(e_{d} \circ e_{u}\right)^{h+1} \circ e_{u}\left(C_{6}\right)$, ce qui donne les quatre valeurs de $d$ :

$$
d_{0}=\frac{v^{2}-1}{v(v-2)}, \quad d_{1}=\frac{-(v-2)}{v^{2}-v+1}, \quad d_{2}=\frac{2 v-1}{v(v+1)}, \quad d_{3}=\frac{v^{2}-v+1}{2 v-1}
$$

REMARQUE 3. On remarque que si on note $g$ l'automorphisme d'ordre 6 de la droite projective défini par

$$
t \mapsto g(t)=\frac{t+1}{2-t}
$$

alors $g^{4}(v)=(v-1) / v$ et on a les résultats suivants : l'application $d \mapsto$ $(d-1) / d$ laisse invariant $d_{0}$, plus précisément $d_{0}=-g(v) g^{4}(v)$ et $d_{0}\left(g^{4}(v)\right)=$ $\left(d_{0}(v)-1\right) / d_{0}(v)$, et elle permute les autres $d_{i}$, plus précisément $d_{2}(v)=$ $\left(d_{1}\left(g^{4}(v)\right)-1\right) / d_{1}\left(g^{4}(v)\right)$ et $d_{3}(v)=-1 /\left(d_{1}(g(v))-1\right)$. 
La famille $E_{d}$ avec $d=d_{1}(z)$ a été donnée dans [4].

Nous obtenons ainsi

THÉORÈme 4 . Les courbes elliptiques $E_{d_{i}}$ avec $i=0,1,2,3$ sont de rang $\geq 1 \operatorname{sur} \mathbb{Q}(v)$ et ht $(d)=2$.

Le point de coordonnées $\left(x_{P_{i}}, y_{P_{i}}\right)$ donné dans le tableau 1 est d'ordre infini.

\section{Tableau 1}

\begin{tabular}{lcc}
\hline & $d$ & $\left(x_{P_{i}}, y_{P_{i}}\right)$ \\
\hline$d_{0}$ & $\frac{v^{2}-1}{v(v-2)}$ & $\left(-\frac{(v-1)(2 v-1)}{v^{2}(v-2)}, \frac{(v-1)^{2}(2 v-1)^{2}}{v^{3}(v-2)^{2}}\right)$ \\
$d_{1}$ & $\frac{-(v-2)}{v^{2}-v+1}$ & $\left(\frac{-v\left(v^{2}-1\right)(v-2)^{2}}{\left.v^{2}-v+1\right)^{3}}, \frac{\left(v^{2}-1\right)^{2}(v-2)^{2}}{\left(v^{2}-v+1\right)^{4}}\right)$ \\
$d_{4}$ & $-6 \frac{z-1}{(z-2)(z-4)}$ & $\left(-9 \frac{(z-1)^{2}}{(z-2)^{2}(z-4)^{2}}, 81 \frac{z(z-1)^{2}}{(z-4)^{3}(z-2)^{4}}\right)$ \\
$d_{5}$ & $\frac{1}{2} \frac{(z+1)(z-4)}{(2 z+1)(z-2)}$ & $\left(-\frac{3}{2} \frac{z(z-1)(z+1)^{2}(z-4)^{2}}{(z+2)^{2}(z-2)^{3}(1+2 z)^{2}}, \frac{9}{8} \frac{z^{2}(z-1)(z+1)^{2}(z-4)^{3}}{(2+z)^{3}(z-2)^{4}(1+2 z)^{3}}\right)$ \\
$d_{6}$ & $8 \frac{w}{w^{3}-w^{2}-w+9}$ & $\left(-2 \frac{w^{2}-9}{w^{3}-w^{2}-w+9}, 4 \frac{\left(w^{2}-9\right)^{2}\left(w^{3}+w^{2}-w-9\right)}{\left(w^{3}-w^{2}-w+9\right)^{3}}\right)$ \\
$d_{7}$ & $-\frac{\left(v^{2}-1\right)(v-2)}{2 v-1}$ & $\left(-\frac{\left(v(v-1)^{2}+1\right)(v+1)\left(v^{2}-v+1\right)\left(v^{3}-v^{2}-1\right)(v-2)^{2}}{(2 v-1)^{4}}\right.$, \\
& & $\left.-\frac{(v+1)\left(v^{3}-v^{2}-1\right)^{2}\left(v(v-1)^{2}+1\right)^{2}(v-2)^{3}}{(2 v-1)^{6}}\right)$ \\
\hline
\end{tabular}

\section{Autres familles de rang 1}

3.1. Exemple 1. On cherche s'il existe des courbes $C$ rationnelles sur $S_{7}$ telles que si $M=(u, v, d) \in C$ alors $e_{d}(M)=(u, v, k / d), k \in \mathbb{Q}$. On obtient de telles courbes avec $k=1$ et $k=1 / 4$ en imposant à la projection de $C$ sur le plan $u=0$ d'être rationnelle. Si $k=1$ on retrouve $d_{0}$. Considérant la courbe correspondant au cas $k=1 / 4$ ainsi que les courbes $\left(e_{d} \circ e_{v}\right)^{h}(C)$ on obtient deux nouvelles valeurs de $d, d_{i}(z), i=4,5$ avec $\operatorname{ht}\left(d_{i}\right)=2$ :

$$
d_{4}(z)=-6 \frac{z-1}{(z-2)(z-4)}, \quad d_{5}(z)=\frac{1}{2} \frac{(z+1)(z-4)}{(2 z+1)(z-2)} .
$$

Les coordonnées d'un point d'ordre infini sur $E_{d_{4}}$ et $E_{d_{5}}$ figurent dans le tableau 1. La courbe $E_{d_{4}}$ possède aussi un point rationnel vérifiant $x=$ $-1 /\left(4 d^{2}\right)[2]$.

Remarque 5. Si $G$ désigne le groupe engendré par les trois involutions on peut construire un sous groupe $\neq \mathrm{Id}$ laissant fixe globalement $C$, correspondant au cas $k=1 / 4$. Si $\varepsilon \in G$ nous n'avons pas obtenu d'exemples de courbe $\varepsilon(C)$ avec ht $(d)<3$. 
3.2. Exemple 2. Considérons la courbe

$$
e_{u}\left(C_{6}\right): \quad\left(u_{1}(v), v_{1}(v)=\frac{v(2 v-1)}{v^{2}-v+1}, d_{1}(v)\right) .
$$

La courbe $H_{v_{1}(v)}$ a un rang sur $\mathbb{Q}(v)$ supérieur ou égal à deux, les points $A_{5}$ et l'image de $e_{u}\left(C_{6}\right)$ sont indépendants. Par combinaison de ces deux points et du point $A_{1}$ d'ordre 4 , on construit comme au paragraphe précédent d'autres familles avec $\mathrm{ht}(d) \geq 3$. Les exemples $d_{6}$ et $d_{7}$ du tableau 1 sont ainsi obtenus. On peut de même utiliser la courbe $\left(u_{4}(z), v_{4}(z)=-\left(z^{2}-4\right) / 3, d_{4}(z)\right)$ de $S_{7}$ et la courbe $H_{v_{4}(z)}$.

\section{Courbes elliptiques de rang $\geq 2$}

4.1. Première méthode. Les égalités $d_{i}(v)=d_{j}(w),\left(d_{i}(w)-1\right) / d_{i}(w)=$ $d_{j}(v)$ et $-1 /\left(d_{i}(w)-1\right)=d_{j}(v)$ définissent des courbes affines et pour chercher des courbes de rang 2 nous chercherons des points rationnels sur ces courbes. Pour $i$ et $j \in\{0,1,2,3,4,5\}$ les courbes obtenues sont des courbes elliptiques sur $\mathbb{Q}$ dont nous allons préciser les équations et le rang sur $\mathbb{Q}$.

Lemme 6. Soit $K$ le corps engendré par les coefficients de la courbe $\Gamma$ d'équation

$$
k(x-a)(x-b)\left(y^{2}-r y+t\right)=\left(y-a_{1}\right)\left(y-b_{1}\right)\left(x^{2}-s x+p\right) .
$$

Il existe un point $\Omega K$-rationnel de $\Gamma$ et une transformation birationnelle $f$ définie sur $K$ telle que $f(\Gamma)$ soit, en général, une courbe elliptique sur $K$ d'élément neutre $f(\Omega)$. Cette courbe elliptique possède un point de torsion $K$-rationnel d'ordre 2 ainsi qu'un point $K$-rationnel. Si de plus les polynômes $x^{2}-s x+p$ et $y^{2}-r y+t$ se factorisent sous la forme $(x-c)(x-d)$ et $\left(y-c_{1}\right)\left(y-d_{1}\right)$ la courbe elliptique possède un autre point $K^{\prime}$-rationnel où $K^{\prime}=K\left(c, d, c_{1}, d_{1}\right)$. Enfin si les fractions rationnelles

$$
h \frac{(x-a)(x-b)}{(x-c)(x-d)}-1 \quad \text { et } \quad m \frac{\left(y-a_{1}\right)\left(y-b_{1}\right)}{\left(y-c_{1}\right)\left(y-d_{1}\right)}-1
$$

avec $h / m=k$ ont des numérateurs qui se factorisent sur $K^{\prime}$ en polynômes de degré 1 en $x$ et en $y$ alors ces courbes elliptiques ont trois points $K^{\prime}$-rationnels généralement indépendants.

Par deux transformations homographiques en $x$ et en $y$ on se ramène à l'étude de

$$
\frac{U}{U^{2}-s U+p}=k \frac{V}{V^{2}-r V+t} .
$$

On pose $U=X\left(X-k^{2} p\right) /(k Y), V=Y /\left(X-k^{2} p\right)$, ce qui définit une application birationnelle d'inverse $X=U V k, Y=V k(V U-k p)$. On obtient alors le modèle de Weierstrass

$$
Y^{2}+Y X(-r+s k)=X(X-t)\left(X-k^{2} p\right) .
$$


Si le discriminant n'est pas nul on obtient une courbe elliptique; le point $p_{1}=(0,0)$ est de 2 -torsion et le point $p_{2}=(t, 0)$ est en général d'ordre infini sur $K$. Le point $p_{3}=\left(k^{2} p, 0\right)$ vérifie $p_{1}+p_{2}+p_{3}=0$. Si les dénominateurs $U^{2}-s U+p$ et $V^{2}-r V+t$ se factorisent en $(U-m)(U-n)$ et $\left(V-m_{1}\right)\left(V-n_{1}\right)$, la courbe en $X, Y$ se factorise aussi sous la forme

$$
\left(Y+X\left(m k-n_{1}\right)\right)\left(Y+X\left(k n-m_{1}\right)\right)=X\left(X-k m m_{1}\right)\left(X-k n n_{1}\right)
$$

et possède les points $K^{\prime}$-rationnels

$$
\begin{array}{ll}
q_{2}=\left(k m m_{1},-m m_{1} k\left(k m-n_{1}\right)\right), & -q_{2}=\left(k m m_{1},-m m_{1} k\left(k n-m_{1}\right)\right), \\
q_{3}=\left(k n n_{1},-n n_{1} k\left(k n-m_{1}\right)\right), & -q_{3}=\left(k n n_{1},-n n_{1} k\left(k m-n_{1}\right)\right) .
\end{array}
$$

On remarque que $q_{3}, q_{2}$ et $p_{1}$ sont alignés, donc $p_{1}+q_{2}+q_{3}=0$.

S'il existe des factorisations de $h \frac{(x-a)(x-b)}{(x-c)(x-d)}-1$ et $m \frac{\left(y-a_{1}\right)\left(y-b_{1}\right)}{\left(y-c_{1}\right)\left(y-d_{1}\right)}-1$ alors on obtient 4 points supplémentaires, ce qui compte tenu des relations évidentes entre les points augmente le rang de 1 au maximum.

Nous regrouperons les résultats obtenus dans le tableau 2. Pour chaque courbe $d_{i}(x)=d_{j}(y)$ nous donnons au moins une valeur de $d=d_{i}\left(x_{1}\right)=$ $d_{j}\left(y_{1}\right)$ telle que la courbe $E_{d}$ soit de rang $\geq 2$, en vérifiant que les points de $E_{d}$ donnés par le tableau 1 sont indépendants.

Tableau 2

\begin{tabular}{lll}
\hline$d_{0}=d_{1}$ & $\begin{array}{l}y^{2}=x^{3}+x^{2}-9 x \\
\operatorname{rang} 1\end{array}$ & $\begin{array}{l}d=15 / 7 \\
\text { conducteur } 2 \cdot 3 \cdot 5 \cdot 7 \cdot 41 \cdot 127\end{array}$ \\
\hline$d_{0}=d_{4}$ & $\begin{array}{l}y^{2}=(x-5)\left(x^{2}+6 x-379\right) \\
\operatorname{rang} 2\end{array}$ & $\begin{array}{l}d=24 / 35 \\
\text { conducteur } 2 \cdot 3 \cdot 5 \cdot 7 \cdot 11 \cdot 13 \cdot 251\end{array}$ \\
\hline \multirow{2}{*}{$=d_{5}$} & $\begin{array}{l}y^{2}=(x-22)\left(x^{2}+23 x-1638\right) \\
\operatorname{rang} 3\end{array}$ & $\begin{array}{l}d=25 / 168 \\
\text { conducteur } \\
2 \cdot 3 \cdot 5 \cdot 7 \cdot 11 \cdot 13 \cdot 29 \cdot 139 \cdot 1847\end{array}$ \\
\hline$d_{5}=\frac{d_{5}-1}{d_{5}}$ & $\begin{array}{l}y^{2}+x y=x^{3}-550315 x+156674225 \\
\operatorname{rang} 3\end{array}$ & $\begin{array}{l}d=-7 / 125 \\
\text { conducteur } 2 \cdot 3 \cdot 5 \cdot 7 \cdot 11 \cdot 1356907\end{array}$ \\
\hline$d_{1}=\frac{-1}{d_{4}-1}$ & $\begin{array}{l}y^{2}=(x+12)\left(x^{2}-11 x+48\right) \\
\operatorname{rang} 1 \text { et 4-torsion }\end{array}$ & $\begin{array}{l}d=21 / 40 \text { et } 80 / 7 \\
\text { rang } 3\end{array}$ \\
\hline$d_{1}=\frac{d_{5}-1}{d_{5}}$ & $\begin{array}{l}y^{2}=(x+17)\left(x^{2}-16 x+87\right) \\
\operatorname{rang} 2\end{array}$ & $\begin{array}{l}d=21 / 2 \\
\text { conducteur } 2 \cdot 3 \cdot 7 \cdot 19 \cdot 2633\end{array}$ \\
\hline$d_{4}=\frac{-1}{d_{5}-1}$ & $\begin{array}{l}y^{2}+x y+y=x^{3}-716 x+182 \\
\operatorname{rang} 2\end{array}$ & $\begin{array}{l}d=-12 / 5 \\
\text { conducteur } 2 \cdot 3 \cdot 5 \cdot 17 \cdot 8863\end{array}$ \\
\hline
\end{tabular}

ThÉORÈme 7. Il existe une infinité de courbes elliptiques sur $\mathbb{Q}$ avec un point rationnel d'ordre 7 et un rang sur $\mathbb{Q}$ supérieur ou égal à 2 . Ces courbes sont paramétrées par les points rationnels de courbes elliptiques. 
Notons $L$ le corps des fonctions sur $\mathbb{Q}$ d'une courbe définie par $d_{i}=d_{j}$. Les résultats du tableau 2 montrent que $E_{d}(L)$ est de rang $\geq 2$ sur $L$. Il résulte d'un résultat de Silverman [6] que les courbes spécialisées $E_{d}$, $d=d_{i}(z)=d_{j}(v),(z, v) \in \mathbb{Q}^{2}$, sont de rang 2 sur $\mathbb{Q}$, sauf peut-être pour un nombre fini de valeurs de $d$.

4.2. Deuxième méthode. Si nous partons d'une famille $E_{d_{i}(t)}$ de courbes de rang 1 sur $\mathbb{Q}(t)$ de générateur $P_{t}$, considérons les points $P_{t}+i A=\left(x_{i}, y_{i}\right)$ où $7 A=0$. Cherchons une condition pour que les points d'ordonnées $y_{i}$ soient rationnels. Pour deux de nos familles $d_{i}$ nous sommes ramenés à chercher des points rationnels sur une courbe elliptique $\mathfrak{C}$ sur $\mathbb{Q}$ de rang positif.

Pour $E_{d_{0}(v)}$, on considère les points de même ordonnée que $P+4 A,-P$ $+A$. La courbe elliptique $\mathfrak{C}$ correspondante est la courbe $y^{2}=x^{3}-12 x+20$ de rang 1 sur $\mathbb{Q}$. On construit ainsi la courbe $E_{35 / 11}$ de petit conducteur $2 \cdot 3 \cdot 5 \cdot 7 \cdot 11 \cdot 13 \cdot 251$, ainsi que $E_{13 / 48}$ de conducteur $2 \cdot 3 \cdot 5 \cdot 7 \cdot 13 \cdot 239 \cdot 827$.

Il en est de même pour la courbe $E_{d_{5}(z)}$ avec le point $P+2 A$. L'une des plus petites valeurs obtenue pour $d$ est $11 \cdot 17 /\left(2^{4} 13\right)$.

5. Courbes de rang $\geq 3$. Divers exemples de courbes de rang 3 ont été trouvés et se répartissent en plusieurs classes.

1) Le premier cas correspond à un point sur les courbes $d_{i}(v)=d_{j}(w)$, $d_{i}(v)=d_{k}(t)$. Chaque égalité définissant une courbe elliptique, ces cas correspondent à des points rationnels sur des courbes de genre $>1$. Par exemple, soit

$$
d=\frac{72}{275}=d_{0}\left(\frac{-11}{7}\right)=d_{5}(-28)=\frac{d_{5}-1}{d_{5}}\left(\frac{32}{23}\right)
$$

et la courbe $E_{d}$

$$
y^{2}+\frac{31 \cdot 41 \cdot 71}{5^{4} 11^{2}} x y+\frac{2^{6} 3^{4} 7 \cdot 29}{5^{6} 11^{3}} y=x^{3}+\frac{2^{6} 3^{4} 7 \cdot 29}{5^{6} 11^{3}} x^{2}
$$

de conducteur $2 \cdot 3 \cdot 5 \cdot 7 \cdot 11 \cdot 29 \cdot 41 \cdot 421 \cdot 2143$. Les points suivants sont indépendants :

$$
\begin{aligned}
& P_{d_{0}}=\left(-\frac{2^{2} 7 \cdot 29}{5^{4} 11}, \frac{2^{2} 7^{2} 13 \cdot 19 \cdot 29^{2}}{5^{8} 11^{3}}\right), \\
& P_{d_{5}}=\left(\frac{2^{6} 3^{4} 7 \cdot 29}{5^{5} 11^{2} 13^{2}},-\frac{2^{14} 3^{8} 7^{2} 29^{2}}{5^{9} 11^{4} 13^{3}}\right), \\
& P_{d_{5}}^{\prime}=\left(-\frac{2^{3} 3^{4} 7 \cdot 23}{5^{5} 11^{3}}, \frac{2^{3} 3^{7} 7^{2}}{5^{7} 11^{4}}\right) .
\end{aligned}
$$

2) La valeur $d=-45 / 11$ est obtenue en spécialisant en $w=-5 / 3$, $t=0$, et $z=10$ les trois fractions rationnelles construites avec les méthodes 
précédemment données, $d_{6}(w), d_{8}(t)=-\frac{3(t+3)(2 t+5)}{(t+1)\left(t^{2}+6 t+11\right)}$ et $\left(d_{5}-1\right) / d_{5}(z)$. Les points

$$
\begin{aligned}
& P_{8}=\left(\frac{3(t+2)(2 t+5)^{2}(t+4)}{\left(t^{2}+6 t+11\right)^{2}(t+1)^{2}}, \frac{18(t+4)^{2}(t+2)(2 t+5)^{3}}{\left(t^{2}+6 t+11\right)^{3}(t+1)^{4}}\right), \\
& P_{5}=\left(\frac{6(z-1) z^{2}(2 z+1)(z-2)}{(z-4)(z+1)^{2}(z+2)^{2}}, \frac{36 z^{4}(z-1)(2 z+1)^{2}(z-2)}{(z-4)^{2}(z+1)^{3}(z+2)^{3}}\right)
\end{aligned}
$$

sont rationnels sur $E_{d}$. Le calcul du régulateur montre qu'ils sont indépendants, ce qui donne la courbe et les points suivants :

$$
\begin{gathered}
y^{2}-\frac{2399}{11^{2}} x y+\frac{2^{3} 3^{4} 5^{2} 7}{11^{3}} y=x^{3}+\frac{2^{3} 3^{4} 5^{2} 7}{11^{3}} x^{2}, \\
P_{6}=\left(\frac{2 \cdot 3 \cdot 7}{11},-\frac{2^{2} 3^{2} 7^{2} 31}{11^{3}}\right), \\
P_{8}=\left(\frac{2^{3} 3 \cdot 5^{2}}{11^{2}}, \frac{2^{6} 3^{2} 5^{3}}{11^{3}}\right), \\
P_{5}=\left(\frac{2 \cdot 3 \cdot 5^{2} 7}{11^{2}}, \frac{2 \cdot 3 \cdot 5^{4} 7^{2}}{11^{3}}\right) .
\end{gathered}
$$

3) La courbe elliptique de rang 3 de plus petit conducteur est sans doute obtenue avec $d=21 / 40$ (voir [2]); son équation est

$$
y^{2}+\frac{1999}{2^{6} 5^{2}} x y+\frac{3^{2} 7^{2} 19}{2^{9} 5^{3}} y=x^{3}+\frac{3^{2} 7^{2} 19}{2^{9} 5^{3}} x^{2}
$$

et son conducteur vaut $2 \cdot 3 \cdot 5 \cdot 7 \cdot 19 \cdot 239 \cdot 419$. Elle possède les points rationnels indépendants

$$
\begin{aligned}
& P_{1}=\left(-\frac{3^{2} 7 \cdot 19}{2^{6} 5^{3}}, \frac{3^{4} 7^{2} 19}{2^{9} 5^{5}}\right), \\
& P_{2}=\left(-\frac{3 \cdot 7^{2}}{2^{3} 5^{3}}, \frac{3 \cdot 7^{4}}{2^{8} 5^{4}}\right), \\
& P_{3}=\left(-\frac{3^{3} 19}{2^{7} 5^{2}}, \frac{3^{6} 19}{2^{13} 5^{3}}\right) .
\end{aligned}
$$

Les deux premiers points correspondent à

$$
\frac{21}{40}=d_{4}(-6) \quad \text { et } \quad \frac{21}{40}=\frac{d_{1}(1 / 8)-1}{d_{1}(1 / 8)} .
$$

Le troisième point ne provient pas d'une famille $d_{n}$ rencontrée dans nos calculs.

Remarque 8. Les calculs de rang ont été faits avec Maple et Apecs, Pari et mwrank (J. Cremona). 


\section{Références}

[1] A. O. L. Atkin and F. Morain, Finding suitable curves for the elliptic curve method of factorization, Math. Comput. 60 (1993), 399-405.

[2] J. Buddenhagen, communication personnelle.

[3] A. Dujella, http://www.math.hr/ duje/tors/tors.html.

[4] L. Kulesz, Arithmétique des courbes algébriques de genre au moins deux, thèse de doctorat, Univ. Paris 7, 1998.

[5] T. Shioda, On the Mordell-Weil lattices, Comment. Math. Univ. St. Paul. 39 (1990), 211-240.

[6] J. Silverman, Heights and specialization map for families of abelian varieties, J. Reine Angew. Math. 342 (1983), 555-565.

[7] J. Stienstra and F. Beukers, On the Picard-Fuchs equation and the formal Brauer group of certain elliptic K3-surfaces, Math. Ann. 271 (1985), 269-304.

Institut de Mathématiques

Université Paris VI

175 rue du Chevaleret

Paris 75013, France

E-mail: lecacheu@math.jussieu.fr

Reçu le 16.5.2001

et révisé le 26.8.2002 